\title{
Factors affecting the normal and branched-chain acyl moieties of teicoplanin components produced by Actinoplanes teichomyceticus
}

\author{
Angelo Borghi, ${ }^{*}$ Duncan Edwards, $\dagger$ Luigi Franco Zerilli and Gian Carlo Lancini
}

MMDRI, Lepetit Research Centre, Via R. Lepetit 34, 21040 Gerenzano (VA), Italy

(Received 6 July 1990; revised 23 October 1990; accepted 13 November 1990)

\begin{abstract}
Teicoplanin, a glycopeptide antibiotic produced by Actinoplanes teichomyceticus, comprises five main components, denoted T-A2-1 to T-A2-5, differing in the structure of their acyl side chain, which is linear in T-A2-1 and T-A2-3 and branched in the other components. Production of T-A2-1, characterized by a linear C10:1 acyl moiety, is entirely dependent on the presence of linoleate in the fermentation medium. Addition to the medium of oleic acid esters at $2 \mathrm{~g} \mathrm{I}^{-1}$ increases the yields of T-A2-3, characterized by a linear C10:0 acyl chain, about threefold. The antibiotic linear side chains thus appear to originate from $\mathrm{C} 18$ unsaturated acid by $\beta$-oxidation degradation. The percentage of T-A2-2, T-A2-4 and T-A2-5, bearing the iso-C10:0, anteiso-C11:0 and iso-C11:0 acyl moieties, respectively, is strongly influenced by the presence in the medium of the amino acids known to be precursors of branched-chain fatty acids. Thus, valine increases the production of T-A2-2 whereas isoleucine or leucine increase the relative yields of T-A2-4 or T-A2-5, respectively. Analysis of the total cell lipids upon addition of the same amino acid shows corresponding increases in the proportion of the iso-C16:0, iso-C15:0 or anteiso-C17:0. A mutant $A$. teichomyceticus strain, which produces a novel teicoplanin with a linear C9:0 chain, differs from the wild strain in the presence of the linear $\mathrm{C17}: 1$ acid in its lipids. The relative incorporation of $\left[{ }^{14} \mathrm{C}\right]$ acetate into teicoplanin acyl moieties is substantially lower when this precursor is added to grown $A$. teichomyceticus mycelium rather than at the time of inoculation. The results suggest that teicoplanin branched acyl moieties also originate from $\beta$-oxidation degradation of cellular long-chain fatty acids.
\end{abstract}

\section{Introduction}

Teicoplanin, a glycopeptide antibiotic recently introduced in clinical practice, is produced by Actinoplanes teichomyceticus as a complex of several active substances (Coronelli et al., 1987). The most interesting ones, originally named teichomycin A-2, were shown (Borghi et al., 1984) to be a complex of at least five closely related factors, designated T-A2-1 to T-A2-5. A sixth active component, teichomycin A-3, not found in fermentation beers, is however always present in crude or purified extracts. It was shown to be a hydrolysis product of the teichomycin A-2 complex (Malabarba et al., 1984).

Teichomycin A-2 components differ in the structure of a fatty acid moiety linked by an amide bond to a glucosamine residue (Barna et al., 1984). As shown in

† Present address: Farmitalia Carlo Erba Research Labs, Via C. Imbonati 24, 20159 Milano, Italy.

Abbreviation: FAME, fatty acid methyl ester.
Fig. 1, the fatty acids are 4-n-decenoic acid (n-C10:1), 8methylnonanoic acid (iso-C10:0), n-decanoic acid (n$\mathrm{Cl0}: 0$ ), 8-methyldecanoic acid (anteiso-C11:0) and 9methyldecanoic acid (iso-C11:0). In addition, four minor components referred to as Related Substances 1 to 4 (RS 1-4) have been recently isolated (Cometti et al., 1988; Borghi et al., 1989). Again they differ in their fatty acid moieties which are 10-methylundecanoic acid (iso$\mathrm{C} 12: 0)$, n-dodecanoic acid (n-C12:0), 6-methyloctanoic acid (anteiso-C9:0) and n-nonanoic acid (n-C9:0), respectively (see Fig. 1).

Although the antibacterial activities of the single components of teicoplanin, determined on a number of clinical isolates, show only minor differences, it was considered desirable to have methods for controlling the composition of batches derived from different fermentations. Therefore, the factors influencing the quantitative composition of the complex in fermentation broths were studied. We report here the results of such studies which suggest a biogenetic relationship between teicoplanin acyl moieties and the fatty acids constituting the lipids of the producing organism. 


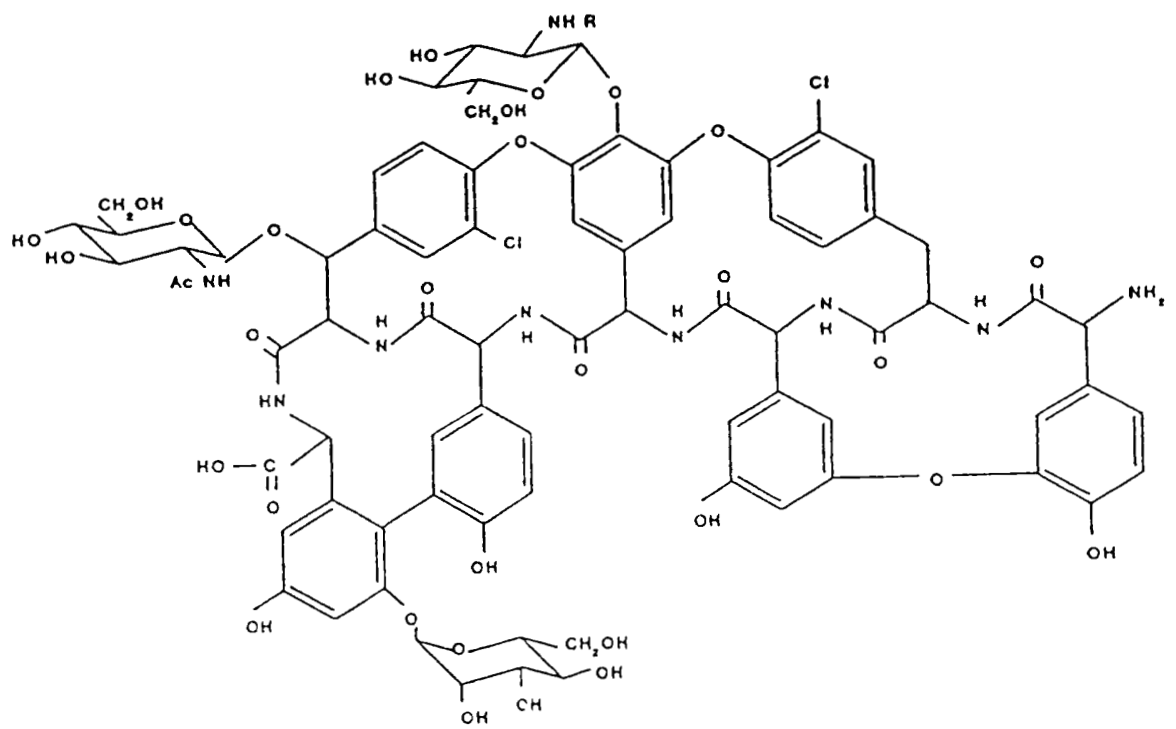

Fig. 1. Chemical structures of the teicoplanins.

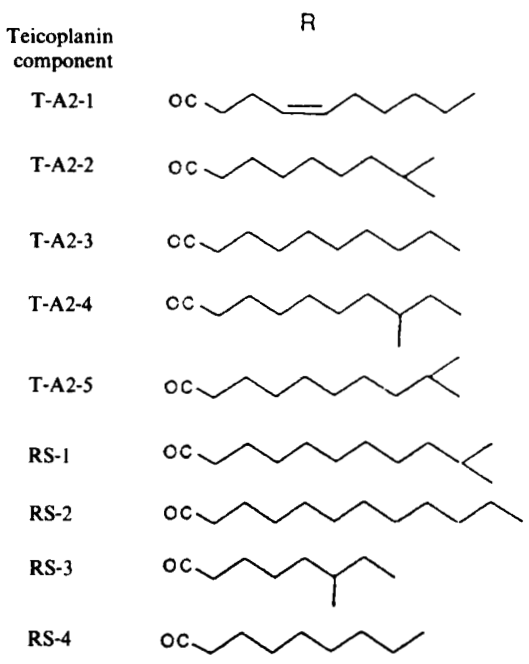

\section{Methods}

Micro-organisms. A. teichomyceticus ATCC 31121 and its mutant derivative A. teichomyceticus A-184 (ATCC 53649), selected after nitrosoguanidine treatment (Borghi et al., 1989), were used.

Culture conditions. A frozen stock culture $(2.5 \mathrm{ml})$ of $A$. teichomyceticus was added as inoculum to a $500 \mathrm{ml}$ Erlenmeyer flask containing $100 \mathrm{ml}$ of seed medium (glucose, $1 \%$; Bacto-peptone (Difco), $0.4 \%$; Bacto yeast extract (Difco), $0.4 \% ; \mathrm{MgSO}_{4} .7 \mathrm{H}_{2} \mathrm{O}, 0.05 \% ; \mathrm{KH}_{2} \mathrm{PO}_{4}$, $0.2 \%$ and $\mathrm{K}_{2} \mathrm{HPO}_{4}, 0.4 \%$ in distilled water). After incubation at $28{ }^{\circ} \mathrm{C}$ for $48 \mathrm{~h}$ on a rotary shaker at 200 r.p.m. ( $5 \mathrm{~cm}$ throw) a $5 \mathrm{ml}$ portion of the seed culture was used to inoculate $100 \mathrm{ml}$ of production medium in a $500 \mathrm{ml}$ Erlenmeyer flask.

Two production media were employed. Medium C comprised glucose, $2 \%$ (sterilized separately); yeast extract $0.5 \%$; asparagine, $0.15 \% ; \mathrm{MgSO}_{4} .7 \mathrm{H}_{2} \mathrm{O}, 0.05 \% ; \quad \mathrm{CaCO}_{3}, 0.5 \% ; \mathrm{NaCl}, \quad 0.01 \%$; $\mathrm{CaCl}_{2} .2 \mathrm{H}_{2} \mathrm{O}, 0.01 \%$; standard mineral solution, $1 \mathrm{ml} \mathrm{1}^{-1}$. Standard mineral solution contained $\left(\mathrm{g} \mathrm{l}^{-1}\right)$ : boric acid, $0.5 ; \mathrm{CuSO}_{4} .5 \mathrm{H}_{2} \mathrm{O}, 0.04$; $\mathrm{KI}, 0.1 ; \mathrm{FeCl}_{3} .6 \mathrm{H}_{2} \mathrm{O}, 0 \cdot 2 ; \mathrm{MnSO}_{4} . \mathrm{H}_{2} \mathrm{O}, 0.4 ; \mathrm{FeSO}_{4} .7 \mathrm{H}_{2} \mathrm{O}, 0.4$; and ammonium molybdate, $\mathbf{0} \cdot 2$. Medium TE/20 comprised yeast extract, $0.6 \%$; maltose, $2 \%$; cotton seed meal, $1.5 \%$; glucose, $2 \%$; and $\mathrm{CaCO}_{3}$, $0.6 \%$.

Production cultures were incubated as for the seed cultures, but usually for $\mathbf{4}$ days.

Isolation and purification of teicoplanin. The culture broth (medium $\mathrm{C}, 100 \mathrm{ml}$ ) was filtered at $\mathrm{pH} 11$ and the mycelium was discarded. The filtered broth was then adjusted to $\mathrm{pH} 7.5$ with $1 \mathrm{M}-\mathrm{HCl}$ and passed through a column containing $20 \mathrm{ml}$ affinity resin (Corti \& Cassani, 1985) equilibrated with $\mathrm{Tris} / \mathrm{HCl}$ buffer $(50 \mathrm{~mm}, \mathrm{pH} 7.5)$. After washing with the same buffer $(40 \mathrm{ml})$, teicoplanin was eluted with ammonia solution $(1 \%, w / v)$ collecting $10 \mathrm{ml}$ fractions. Fractions containing teicoplanin, neutralized with $2 \mathrm{M}-\mathrm{HCl}$, were combined and passed through a desalting column containing silanized silica gel (10 ml, Merck 7719) equilibrated with water. The column was washed with water $(20 \mathrm{ml})$ and eluted with $\mathrm{H}_{2} \mathrm{O} /$ acetonitrile $(1: 1, \mathrm{v} / \mathrm{v})$. Fractions ( $5 \mathrm{ml}$ each) containing teicoplanin were combined, concentrated under a gentle stream of nitrogen and lyophilized.
Analysis of fatty acids. Mycelium grown in medium $\mathrm{C}$ was recovered by centrifugation, washed with water and freeze-dried. For analysis of whole-cell fatty acids, a portion ( $15 \mathrm{mg}$ ) was saponified with $1 \mathrm{M}-\mathrm{NaOH}$ in methanol/toluene $(70: 30, \mathrm{v} / \mathrm{v} ; 3 \mathrm{ml})$ at $100^{\circ} \mathrm{C}$ for $30 \mathrm{~min}$. Acidification with gaseous $\mathrm{HCl}$ dissolved in anhydrous methanol to give $2 \mathrm{M}$ final concentration $\left(3 \mathrm{ml}, 30 \mathrm{~min}\right.$ at $\left.85^{\circ} \mathrm{C}\right)$, and addition of $14 \%(\mathrm{w} / \mathrm{v})$ boron trifluoride/methanol reagent $\left(1 \mathrm{ml}, 10 \mathrm{~min}\right.$ at $\left.100^{\circ} \mathrm{C}\right)$ (Supelco BF 3 -methanol Kit 3-3020), after filtration, allowed conversion of fatty acids to their methyl esters (FAMEs). FAMEs were extracted in hexane $(3 \mathrm{ml}$, twice) and the solvent was concentrated to $0.25 \mathrm{ml}$. Gas chromatography-mass spectrometry (GC-MS) analysis of FAMEs was carried out on a Hewlett-Packard HP 5985-B instrument. Hexane solution $\left(1 \mu \mathrm{l}\right.$ ) was injected (at $200^{\circ} \mathrm{C}$, split ratio $1 / 10$ ) onto a HewlettPackard high-performance fused silica capillary column (internal diameter $0.3 \mathrm{~mm}$, length $25 \mathrm{~m}$ ). The following methods were used: method 1: column coated with a $0.5 \mu \mathrm{m}$ thick film of $5 \%$ cross-linked phenyl-methylsilicone; temperature programme $120^{\circ} \mathrm{C}$ to $230{ }^{\circ} \mathrm{C}$ at $3^{\circ} \mathrm{C}$ min $^{-1}$; column head pressure 10 p.s.i. $(69 \mathrm{kPa})$; method 2 : dimethylsilicone fluid coated column; temperature programme $80^{\circ} \mathrm{C}$ for $1 \mathrm{~min} ; 8^{\circ} \mathrm{C} \mathrm{min}^{-1}$ to $250^{\circ} \mathrm{C}$; column head pressure 7.5 p.s.i. $(52 \mathrm{kPa})$.

For specific analysis of fatty acid content in phospholipids, the lipids were extracted according to the method of Lechevalier et al. (1977). The crude extract was then saponified and derivatized to obtain FAMEs as described above for whole-cell fatty acids.

Incorporation of labelled acetate. Mycelium was grown in medium $\mathrm{C}$ $(100 \mathrm{ml})$ for $30 \mathrm{~h}$ then washed and resuspended in sterile $0.1 \% \mathrm{NaCl}$ solution $(100 \mathrm{ml})$. Sodium $\left[2-{ }^{14} \mathrm{C}\right]$ acetate (specific activity $53 \mathrm{mCi}$ $\mathrm{mmol}^{-1} ; 1961 \mathrm{MBq} \mathrm{mmol}{ }^{-1}$ Amersham) was added either to medium $C$ before inoculation or to the mycelium resuspended in saline. Fermentation was carried out for $24 \mathrm{~h}$ at $28^{\circ} \mathrm{C}$ on a rotary shaker at 200 r.p.m.

Hydrolysis of teicoplanin. Hydrolysis of teicoplanin to give its pseudoaglycone (T-A3-1) was carried out by the method of Strazzolini et al. (1986). Teicoplanin (about $4 \mathrm{mg}$ ) was dissolved in $90 \%(\mathrm{v} / \mathrm{v})$ trifluoroacetic acid $(0.4 \mathrm{ml})$ and kept for $1.5 \mathrm{~h}$ at room temperature. The sample was then evaporated to dryness under $\mathrm{N}_{2}$ and the residue dissolved in water/methanol $(1: 1, \mathrm{v} / \mathrm{v} ; 0.4 \mathrm{ml})$. The solution $(10-20 \mu \mathrm{l})$ was applied as a narrow start band on a RP-8 $F_{254}$ S $0.25 \mathrm{~mm}$ precoated 
plate (Merck 15682) and developed with a $\mathrm{Na}_{2} \mathrm{SO}_{4}(5 \% \mathrm{w} / \mathrm{v}$, aqueous solution)/acetonitrile $(7: 3 \mathrm{v} / \mathrm{v})$. The spots corresponding to T-A3-1 ( $254 \mathrm{~nm}$ detection) and to the acylglucosamines (iodine vapours) were separately scraped off, eluted from the adsorbent with water/methanol $(1: 1, \mathrm{v} / \mathrm{v})$ and their radioactivity measured.

Analytical methods. For high-performance liquid chromatography (HPLC), a Waters instrument with a Zorbax ODS (Du Pont) column $(4.6 \times 150 \mathrm{~mm})$ was used. A gradient of $8 \%$ to $40 \%$ phase B $(0.02 \mathrm{M}-$ $\mathrm{NaH}_{2} \mathrm{PO}_{4} /$ acetonitrile, $\left.95: 5, \mathrm{v} / \mathrm{v}\right)$ in phase A $\left(0.02 \mathrm{M}-\mathrm{NaH}_{2} \mathrm{PO}_{4} /\right.$ acetonitrile, $25: 75, \mathrm{v} / \mathrm{v}$ ) required $40 \mathrm{~min}$ at a flow rate of $1.5 \mathrm{ml} /$ $\min ^{-1}$. The detector was set at $254 \mathrm{~nm}$.

Radioactivity was measured with a Packard Tri-Carb $460 \mathrm{C}$ automatic liquid scintillation system using Insta-Gel (Packard) counting cocktail.

\section{Results}

Preliminary experiments indicated that the relative amounts of teicoplanin components were influenced by the composition of the fermentation medium. This was especially evident for the components bearing linear fatty acids, which were enhanced by addition of fats or oils. As shown in Fig. 2, in which the HPLC profiles of teicoplanin produced under two different conditions are shown, the presence of component T-A2-1 depended on the medium composition; T-A2-1 was absent when the only nutrients were glucose and yeast extract (medium C). The determining factor for production of T-A2-1 was the presence of esters of linoleic acid in the cotton seed meal used as a nitrogen source in medium TE/20.

Typical results of systematic series of experiments (Table 1) clearly indicate that T-A2-1 is preferentially produced in the presence of trilinolein and T-A2-3 in the presence of triolein. Similar results were obtained using methyl or ethyl linoleate or oleate respectively. It is noteworthy (results not shown) that no change in total yields or in the relative amounts of components was observed when esters of saturated fatty acids such as
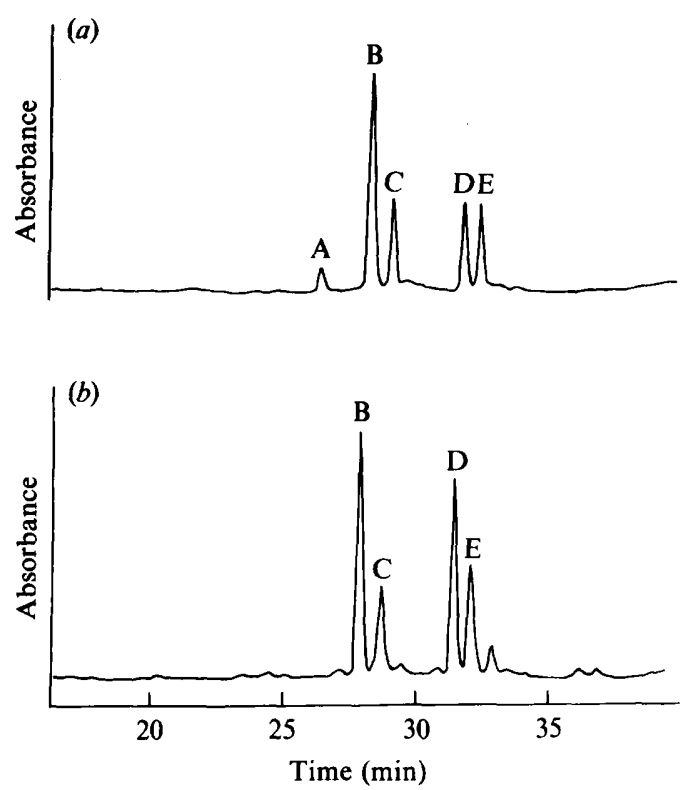

Fig. 2. HPLC profiles of the teicoplanin complexes produced in: (a) medium Te/20; and (b) medium C. A, B, C, D and E represent T-A2-1, T-A2-2, T-A2-3, T-A2-4 and T-A2-5, respectively.

stearate or palmitate were added to the fermentation medium.

Altogether, these results suggest that biosynthesis of the acyl moieties is a limiting step in the production of individual teicoplanin components and that the two linear ten-carbon acids derive, probably through the usual $\beta$-oxidation mechanism, from the corresponding eighteen-carbon unsaturated acids. The position of the double bond in the 4-decenoic acid corresponds to that of the double bond in position 12 of linoleic acid, assuming the loss of four acetate units by $\beta$-oxidation degradation.

A selective increase of the relative amounts of components T-A2-2, T-A2-4 and T-A2-5 by specifically stimulating the biosynthesis of their characteristic acyl moieties was then attempted.

Table 1. Effect of biosynthetic precursors on teicoplanin complex composition

Cultures were grown in medium C. Precursors were added $24 \mathrm{~h}$ after the inoculum. The results are the means of three flasks.

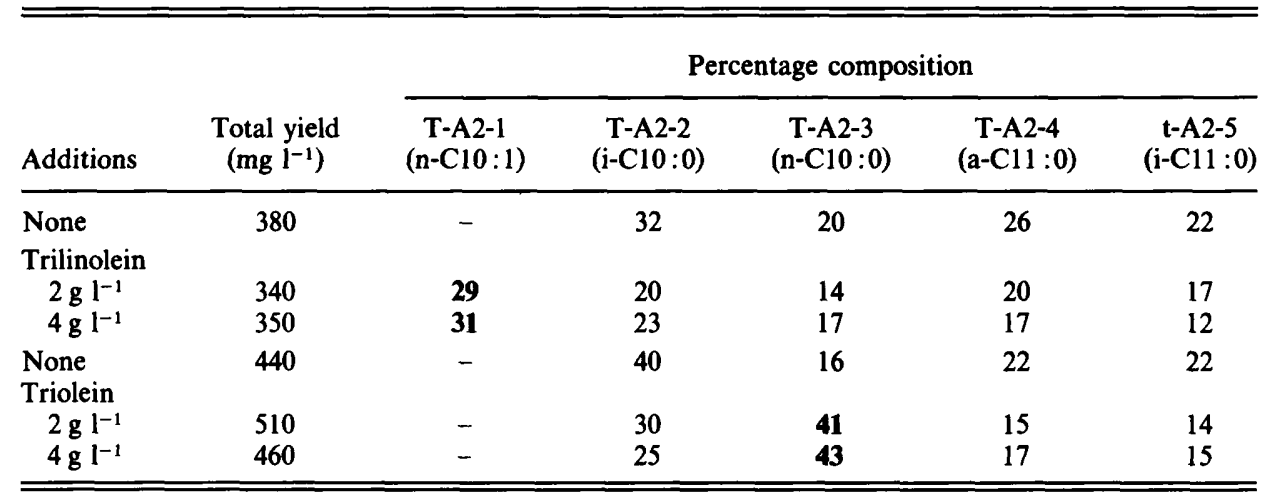


Stimulation of branched fatty acid synthesis can generally be obtained by supplying a specific precursor, since it is known (Kaneda, 1977) that isobutyrate, isovalerate and 2-methylbutyrate are the starting molecules of the polymerization process leading to evencarbon iso acids, odd-carbon iso acids and odd-carbon anteiso acids, respectively. Since butyric or valeric acids themselves are often toxic to micro-organisms they can be supplied as esters, or indirectly by supplying valine, leucine or isoleucine from which they are generated by common metabolic pathways.

Addition of valine substantially increased both the relative and absolute yields of component $\mathrm{T}-\mathrm{A} 2-2$, characterized by the iso-C10:0 moiety (Table 2). Similar increases were observed in the amounts of T-A2-4 (bearing anteiso-C11:0) with isoleucine, and of T-A2-5 (bearing iso-C11:0) with leucine. These results could be interpreted as evidence for a direct synthesis of these acids by an antibiotic-specific synthase. However, it is unlikely that there are two different pathways (direct synthesis and degradation of longer chains) for the branched and linear fatty acid moieties of the same antibiotic. Thus we looked for the presence of possible long chain precursors of the branched fatty acids.

Most cellular fatty acids in Gram-positive bacteria are constituents of membrane lipids. Branched fatty acids are typical membrane constituents of actinomycetes (Kroppensted, 1985), and certain other micro-organisms. GC-MS of the total fatty acids of $A$. teichomyceticus gave the pattern shown in Fig. 3. Since superimposable results were obtained when the same analysis was carried out with extracted cellular phospholipids we assume that this pattern refiects the composition of the cell membrane fatty acids. The major peaks corresponded to 14methylpentadecanoic acid (iso-C16:0), 13-methyltetradecanoic acid (iso-C15:0) and 14-methylhexadecanoic

Table 2. Effect of biosynthetic precursors on teicoplanin complex composition

Cultures were grown in medium C. Precursors were added $24 \mathrm{~h}$ after the inoculum. The results are the means of three flasks.

\begin{tabular}{|c|c|c|c|c|c|}
\hline \multirow[b]{2}{*}{ Additions } & \multirow[b]{2}{*}{$\begin{array}{l}\text { Total yield } \\
\left(\mathrm{mg} \mathrm{i}^{-1}\right)\end{array}$} & \multicolumn{4}{|c|}{ Percentage composition } \\
\hline & & $\begin{array}{c}\text { T-A2-2 } \\
(\mathrm{i}-\mathrm{C} 10: 0)\end{array}$ & $\begin{array}{c}\text { T-A2-3 } \\
\text { (n-C10:0) }\end{array}$ & $\begin{array}{c}\text { T-A2-4 } \\
(\mathrm{a}-\mathrm{C} 11: 0)\end{array}$ & $\begin{array}{c}\text { T-A2-5 } \\
\text { (i-C11:0) }\end{array}$ \\
\hline None & 340 & 34 & 20 & 24 & 21 \\
\hline $\begin{array}{r}\text { L-Valine } \\
1 \mathrm{~g} \mathrm{l}^{-1} \\
2 \mathrm{~g} \mathrm{l}^{-1}\end{array}$ & $\begin{array}{l}650 \\
790\end{array}$ & $\begin{array}{l}70 \\
83\end{array}$ & $\begin{array}{r}12 \\
9\end{array}$ & $\begin{array}{r}10 \\
4\end{array}$ & $\begin{array}{l}7 \\
3\end{array}$ \\
\hline $\begin{array}{l}\text { L-Isoleucine } \\
1 \mathrm{~g} \mathrm{l}^{-1} \\
\mathrm{~L}-\mathrm{Leucine} \\
\operatorname{l\mathrm {g}\mathrm {I}^{-1}}\end{array}$ & 130 & 24 & 13 & 40 & 20 \\
\hline
\end{tabular}
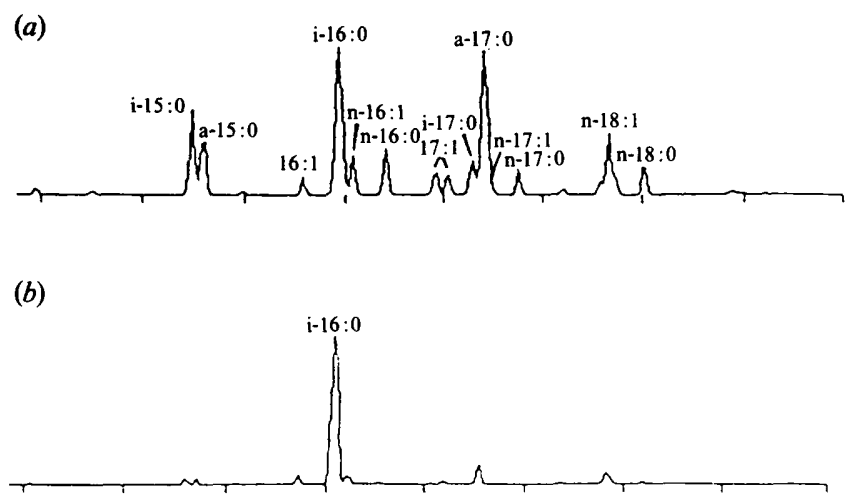

(c)
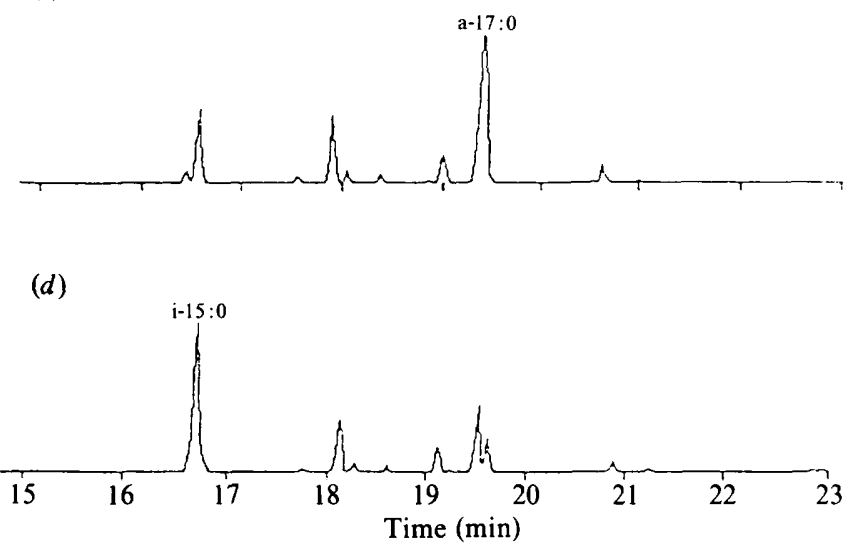

Fig. 3. FAME GC-MS profiles for A. teichomyceticus strain 44 wholecell hydrolysates from fermentations: $(a)$ unsupplemented (see also Fig. 4); (b) with added valine; $(c)$ with added isoleucine; $(d)$ with added leucine. The amino acids were added to medium $C$ at a final concentration of $1 \mathrm{~g} \mathrm{I}^{-1} 24 \mathrm{~h}$ after inoculation. Chromatographic method 1 was used.

acid (anteiso-C17:0), which are logical precursors of the iso-C10:0, iso-C11:0 and anteiso-C11:0 moieties of TA2-2, T-A2-5 and T-A2-4, respectively. In addition, oleic acid (n-C18:1), the precursor of the $\mathrm{n}-\mathrm{C} 10: 0$ moiety, was present whereas linoleic acid (n-C18:2) was absent, in agreement with the observation that the decenoic moiety of T-A2-1 is produced only when linoleic acid is supplied to the fermentation medium.

Addition to the cultures of the amino acid precursors of the branched fatty acids markedly modified the lipid composition. As shown in Fig. 3, when valine $\left(1 \mathrm{~g} \mathrm{l}^{-1}\right)$ was added, iso-C16:0 was almost the only component of the cellular fatty acids. Superimposable results were obtained when the analysis was repeated on extracted lipids. Upon addition of leucine or isoleucine, iso-C15:0 or the anteiso-C17:0, respectively, became the major constituents. This exactly paralleled the increase in teicoplanin components T-A2-2, T-A2-5 and T-A2-4 described above and suggests, although it does not prove, 
a biosynthetic relationship between the antibiotic fatty acid moieties and the cellular lipids.

Further support for this hypothesis came from the isolation of $A$. teichomyceticus mutant A-184, morphologically indistinguishable from the parent strain, but producing substantial amounts of two teicoplanin analogues, RS-3 and RS-4, having an iso-C9:0 and a n-C9:0 chain, respectively (Borghi et al., 1989). The presence of the iso derivative could easily be explained by a further loss of acetate from the iso- $\mathrm{Cl1}: 0$ deriving from iso$\mathrm{C} 15: 0$. The linear nonanoic acid should derive from a linear odd-carbon acid, probably unsaturated because saturated linear acids were shown not to be utilized as precursors. The major difference between the mutant and parent strain fatty acids was indeed, as shown in Fig. 4 , the presence of a substantial amount of linear heptadecenoic acid, as well as the presence of $n$ pentadecanoic and $n$-heptadecanoic acids.

The final demonstration that cell fatty acids are precursors of antibiotic fatty acid moieties was provided by experiments with labelled acetate. A. teichomyceticus was grown in medium $\mathrm{C}$ for $30 \mathrm{~h}$. At this time, when antibiotic production had just started, the mycelium was filtered, washed and resuspended in buffer. After a further $24 \mathrm{~h}$, teicoplanin was isolated and hydrolysed as shown in Fig. 5.

When $\left[2-{ }^{14} \mathrm{C}\right]$ acetate was added to the growth medium, $36.4 \mathrm{mg}$ of $\left[{ }^{14} \mathrm{C}\right]$ teicoplanin with a specific activity of 38850 d.p.m mg $\mathrm{m}^{-1}$ was obtained. After hydrolysis,
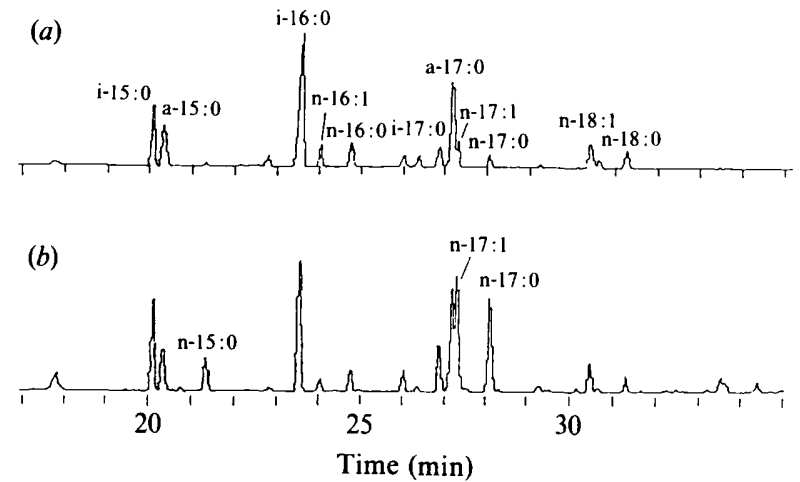

Fig. 4. FAME GC-MS profiles for A. teichomyceticus whole-cell hydrolysates from: (a) strain 44 (as in Fig. 3); (b) mutant strain A-184. Chromatographic method 2 was used.

$78 \%$ of the antibiotic radioactivity was found in the pseudoaglycone and $22 \%$ in the acylglucosamine moiety. This result is consistent with an approximately uniform distribution of radioactivity in the carbon atoms deriving from carbon 2 of acetate. On the basis of what is known about the biosynthesis of glycopeptide antibiotics (Hammond et al., 1983; Chung et al., 1986), nine carbon atoms in the pseudoaglycone and three in each acid chain are derived from carbon 2 of acetate. When $\left[2-{ }^{14} \mathrm{C}\right]$ acetate was added to the resuspended mycelium, $48.9 \mathrm{mg}$ of teicoplanin with a specific activity of 159000 d.p.m. $\mathrm{mg}^{-1}$ was obtained. After hydrolysis, only $4.8 \%$ of the radioactivity was associated with the acylglucosamine,

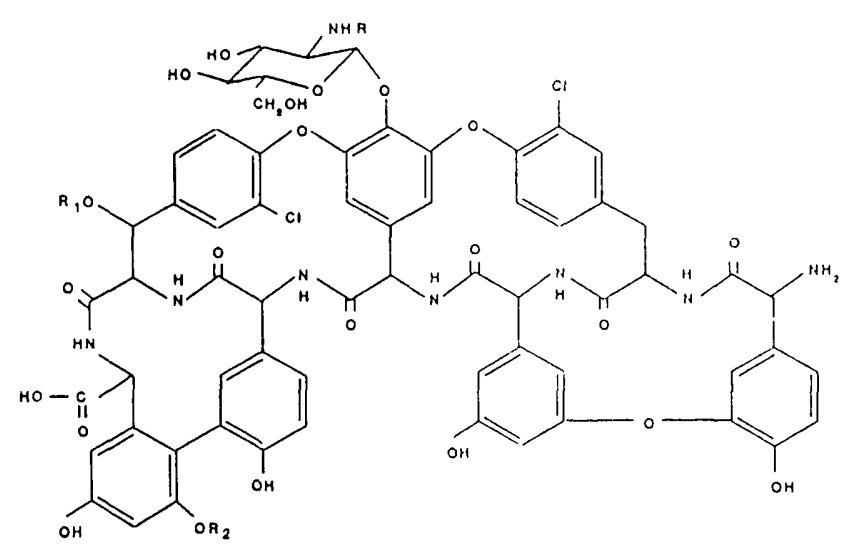

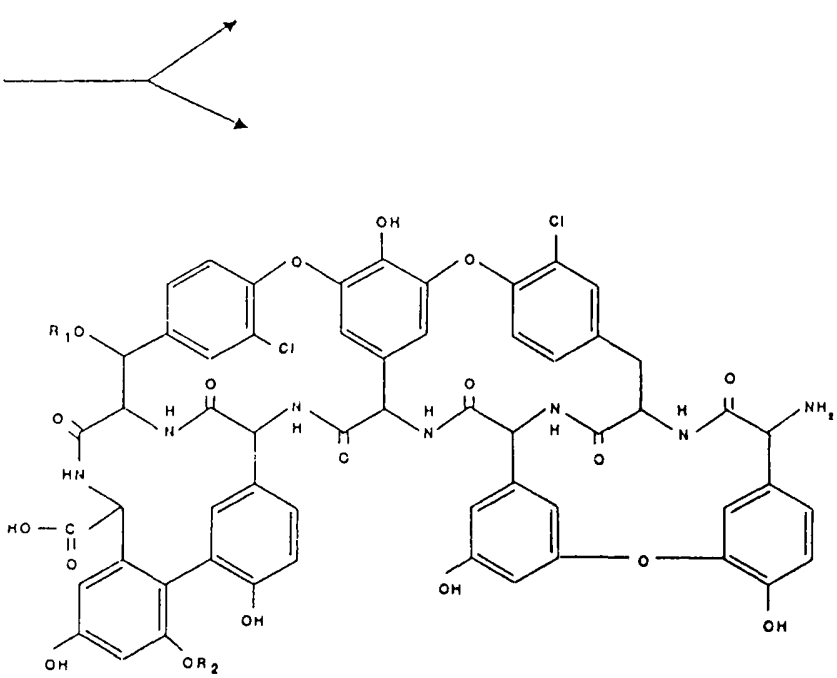

Fig. 5. Hydrolysis of T-A2-2 to T-A3-1 and $N$-(8-methylnonanoyl)glucosamine. $R=8$-methylnonanoyl; $R_{1}=N$-acetyl- $\beta$-glucosaminyl; $\mathbf{R}_{2}=\alpha$-D-mannosyl. 
demonstrating that the fatty acid moieties must have come from molecules formed during the growth phase.

\section{Discussion}

The results show that teicoplanin acyl moieties originate by degradation of fatty acids from the cell membrane or, in the case of linoleic acid, present in the fermentation medium.

This is supported by: (a) the absolute requirement for linoleic acid in the fermentation medium to obtain T-A2$1 ;(b)$ the strong increase in the production of T-A2-3 upon addition of oleic acid to the medium; $(c)$ the consistent relationship between the amount of a given cellular acid and the yield of the corresponding component of the teicoplanin complex, both in the presence and in the absence of specific precursors; $(d)$ the presence in a mutant strain producing a component with a linear odd carbon acyl moiety of an unsaturated linear acid with seventeen carbon atoms; $(e)$ the low incorporation of labelled acetate in the acyl moieties when this precursor was added to cultures after completion of mycelial growth. This last result is in agreement with the report (Chung et al., 1986) that $\left[{ }^{14} \mathrm{C}\right]$ oleate, but not $\left[{ }^{14} \mathrm{C}\right]$ acetate labelled the linear fatty acid moiety of ardacins (aridicins) when added to a fermentation after growth was complete.

The change in cellular fatty acid composition caused by precursor amino acids is of interest. Since similar results were obtained by analysing cell phospholipids, the membrane composition was altered in a parallel manner. This result is in agreement with previously reported observations on the effect of amino acids on the lipid composition of several Bacillus species (Kaneda, 1977) and of Streptomyces hygroscopicus and Streptomyces griseus (Graefe et al., 1982a). However, the extent of the alteration caused by a moderate amount of valine is surprising.

Alteration in fatty acid content due to genetic changes in Streptomyces have been reported previously: Arima et al. (1973) described a mutant $S$. fradiae strain with an altered ratio of anteiso-C15:0 to iso-C16:0. Similarly (Graefe et al., 1982a,b) reported an altered ratio of anteiso-C15:0 to iso-C16:0 in Streptomyces mutants defective in the production of aerial mycelium. Mutant A-184 of $A$. teichomyceticus, although almost indistinguishable from the parent strain in morphology and growth characteristics, is significantly different in lipid composition, which includes substantial amounts of oddcarbon linear fatty acids virtually undetectable in the parent strain.

The pattern of fatty acids in cell lipids has been proposed and used as an element for taxonomic classification of bacteria (Kroppensted, 1985). Our results suggest that this criterion should be used with care since variations are possible, even within the same species.

The authors are indebted to Dr A. Gianantonio and Dr F. Assi for providing initial data on the effect of fatty acids in teicoplanin fermentation, and wish to thank Dr G. G. Gallo for useful discussion.

\section{References}

ARima, K., OKazaki, H., Ono, H., Yamada, K. \& BepPU, T. (1973). Effect of exogenous fatty acids on the cellular fatty acids composition and neomycin formation in a mutant strain of Streptomyces fradiae. Agricultural and Biological Chemistry 37, 2313-2317.

Barna, J. C., Williams, D. H., Stone, D. J. M., Leung T. W. C. \& DoDDReLl, D. M. (1984). Structure elucidation of the teicoplanin antibiotics. Journal of the American Chemical Society 106, 4895-4902.

Borghi, A., Coronelli, C., Fanivolo, L., Allievi, G., Pallanza, R. \& Gallo, G. G. (1984). Teichomycins, new antibiotics from Actinoplanes teichomyceticus nov. sp. IV. Separation and characterization of the components of teichomycin (teicoplanin). Journal of Antibiotics, 37, 615-620.

Borghi, A., Antonini, P., Zanol, M., Ferrari, P., Zerilli, L. F. \& LANCINI, G. C. (1989). Isolation and structure determination of other two related substances of teicoplanin, a glycopeptide antibiotic. Journal of Antibiotics 42, 361-366.

Chung, S. K., Taylor, P., OH, Y. K., DeBrosse, C. \& Jeff, P. W. (1986). Biosynthetic studies of aridicin antibiotics. I. Labelling pattern and overall pathways. Journal of Antibiotics 39, 642-651.

Cometti, A., Gallo, G. G., Kettenring, J., Panzone, G. B., Tuan, G. \& ZERILLI, L. F. (1988). Isolation and structure determination of the main related substances of teicoplanin, a glycopeptide antibiotic. Il Farmaco Edizione Scientifica 43, 1005-1018.

Coronelli, C., Gallo G. G. \& Cavalleri, B. (1987). Teicoplanin: chemical, physico-chemical and biological aspects. Il Farmaco Edizione Scientifica 42, 767-787.

CORTI, A. \& CASSANI, G. (1985). Synthesis and characterization of Dalanyl-D-alanine-agarose. Applied Biochemistry and Biotechnology 11, 101-108.

Graefe, U., Roth, M. \& KREBS, D. (1982a). Effect of L-valine and Lisoleucine on fatty acid composition of Streptomyces hygroscopicus and S. griseus. Zeitschrift für Allgemeine Mikrobiologie 22, 595-599.

Graefe, U., Reinhardt, G., Krebs, D., Roth, M. \& Noack, D. $(1982 b)$. Altered lipid composition in a non-differentiating derivative of Streptomyces hygroscopicus. Journal of General Microbiology 128, 2693-2698.

Hammond, S. J., Williams, D. H. \& Nielsen, R. V. (1983). The biosynthesis of ristocetin. Journal of the Chemical Society Chemical Communications, 116-117.

KANEDA, T. (1977). Fatty acids of the genus Bacillus: an example of branched chain preference. Bacteriological Reviews 41, 391-418.

KROPPENSTED, R. M. (1985). Fatty acids and menaquinone analysis of actinomycetes and related organisms. In Chemical Methods in Bacterial Systematics pp. 173-199. Edited by M. Goodfellow \& D. E. Minnikin. London: Academic Press.

Lechevalier, M. P., De Bievre, C. \& Lechevalier, H. (1977). Chemotaxonomy of aerobic actinomycetes: phospholipid composition. Biochemical Systematics and Ecology 5, 249-260.

Malabarba, A., Strazzolini, P., Depaoli, A., Landi, M., Berti, M. \& CAVAlleRI, B. (1984). Teicoplanin, antibiotics from Actinoplanes teichomyceticus nov. sp. VI. Chemical degradation: physico-chemical and biological properties of acid hydrolysis products. Journal of Antibiotics 37, 988-999.

Strazzolini, P., Malabarba, A. \& Cavaller1, B. (1986). Chemical process for preparing antibiotic L17054. US Patent 4594187. 